\title{
Etalement Urbain Et Tensions Foncieres Dans Les Villages Peripheriques De Daloa (Centre-Ouest, Cote d'Ivoire)
}

\author{
Gouamene Didier Charles \\ Enseignant-Chercheur \\ Université Jean Lorougnon Guédé (Daloa, Côte d'Ivoire) \\ Oura Kouadio Raphael \\ Université Alassane Ouattara (Bouaké, Côte d'Ivoire) \\ Centre de Recherche pour le Développement \\ Ouattara Sahoti \\ Enseignant-Chercheur \\ Université Jean Lorougnon Guédé (Daloa, Côte d'Ivoire)
}

Doi: 10.19044/esj.2017.v13n35p217 URL:http://dx.doi.org/10.19044/esj.2017.v13n35p217

\begin{abstract}
The city of Daloa, located in the Ivorian west-centre, is experiencing a rapid spatial growth in recent years due to the acceleration of urbanization. This phenomenon, which should logically constitute an opportunity, because of the consumer market that it represents for the peri-urban villages, is becoming one of the causes of the tensions, then weakening social cohesion. The current urban dynamics cause the problem of space occupation by buildings and the destruction of natural and land resources around the city. Faced with this urban sprawl, which is increasingly aggressive and capable of swallowing up all the surrounding rural areas, the village communities express their concern over the transformation of their regions. In this framework, where the fear of the future has settled in the countryside and where land has become a major issue, the resulting competition for land is more than ever strengthened. This has resulted in land tensions that undermine their resilience faced with the scale of urbanization.

The study, which is based on the dual quantitative and qualitative approach, aims to understand both the link between land tensions and urban spread and the perception and strategies developed by rural populations faced with urban pressure.
\end{abstract}

Keywords: Daloa; urban spread; land tensions; perception; strategies 


\section{Résumé}

La ville de Daloa, dans le centre-ouest ivoirien, connaît ces dernières années une croissance spatiale rapide due à l'accélération de l'urbanisation. Ce phénomène qui devait logiquement constituer une opportunité, de par le marché de consommation qu'elle représente pour les villages périurbains, est en passe de devenir pour ceux-ci, l'une des causes des tensions qui fragilisent aujourd'hui la cohésion sociale. La dynamique urbaine qui a cours, pose en effet le problème de l'occupation de l'espace par le bâti et de la destruction des ressources naturelles et foncières aux alentours de la ville. Face à cet étalement urbain de plus en plus agressif et capable de phagocyter l'entièreté des territoires ruraux environnants, les communautés villageoises expriment leur inquiétude devant la transformation de leurs terroirs. Dans ce contexte où la peur de l'avenir s'est installée dans les campagnes et où la terre est devenue un enjeu majeur, la compétition foncière qui en découle s'en trouve plus que jamais renforcée. Il en est résulté des tensions foncières qui mettent à mal leur capacité de résilience face à l'ampleur de l'urbanisation.

L'étude qui a reposé sur la double approche quantitative et qualitative, a donc pour objectif de chercher à comprendre aussi bien le lien entre les tensions foncières et l'étalement urbain que la perception et les stratégies développées par les populations rurales face à la poussée urbaine.

Mots-clés : Daloa ; étalement urbain; tensions foncières ; perception ; stratégies

\section{Introduction}

Faiblement urbanisée au début des années 1960 (6\% à 7\% par an), l'Afrique connait aujourd'hui une explosion urbaine (Laborde, 2005). En Côte d'Ivoire, la rapidité du taux d'urbanisation s'est traduite par l'étalement de nombreuses villes dont Daloa.

Ville du centre-ouest ivoirien, elle connait une évolution spatiale rapide due à une forte croissance démographique depuis plus de 50 ans (Alla, 1991). La dynamique urbaine qui a cours incorpore dans son giron les espaces naturels et agricoles aux alentours de la ville. Avec une superficie de 217,75 ha et une population de 7487 habitants dans les années 1950, la surface urbaine de Daloa est estimée aujourd'hui à 3300 ha pour une population de 255354 habitants (Koukougnon, 2012 ; INS, 2014). Cette extension consécutive à la surconsommation foncière a non seulement réduit les aires d'extension des villages environnants ; mais aussi provoqué des changements dans les rapports de l'homme à la terre. Désormais, cette ressource est au centre de divers enjeux ; elle est devenue un bien rare et disputé par les multiples acteurs présents sur la scène foncière (Paulet, 2000). 
Face à la situation qui prévaut, les communautés villageoises expriment leur inquiétude devant la disparition de leurs finages. La compétition née de ces enjeux a fait naitre de vives tensions entre acteurs.

La présente contribution a pour objectif principal de chercher à comprendre aussi bien le lien entre les tensions foncières et l'étalement urbain que la perception et les stratégies développées par les populations rurales pour répondre à l'avancée de la ville. De manière spécifique, il s'agit d'abord de présenter les caractéristiques de l'étalement urbain à Daloa. Ensuite, d'identifier les tensions foncières nées de l'étalement de la ville de Daloa. Enfin, d'analyser la perception et les stratégies développées par les populations face au phénomène.

\section{Approche methodologique}

\section{Présentation de la zone d'étude}

Située dans la région du Haut-Sassandra au Centre-Ouest de la Côte d'Ivoire, la ville de Daloa s'étend sur 3300 ha et compte une population de 255354 habitants (RGPH, 2014). Limitée au Nord par les départements de Vavoua et de Zuenoula, au Sud par ceux d'Issia et de Sinfra, à l'Ouest par les départements de Duékoué et de Bangolo et à l'Est par celui de Bouaflé, la ville de Daloa a été érigée en commune depuis 1980. Née de la fusion des villages (Lobia, Labia, Gbeuliville et Tazibouo) en bordure des pistes NordSud et Est-Ouest, Daloa dont l'évolution s'est faite autour desdits villages, est avant tout le territoire des populations d'autochtones Bété. L'ethnie Bété fait partie du sous-groupe culturel Krou, une société à dominance patrilinéaire. La gestion foncière chez les Bété revient à l'aîné de chaque groupe familial qui, du reste, fait office de chef de terre. Il est le seul habilité à procéder à la distribution de la terre à chaque membre de la famille. Du fait de son extension, la commune compte aujourd'hui une quarantaine de quartiers et une douzaine de villages-quartiers dont Sapia, Zaguiguia, Balouzon, Gbokora, Zakoua, etc. (figure ${ }^{\circ} 1$ ). 
Figure 1 : Espace d'étude

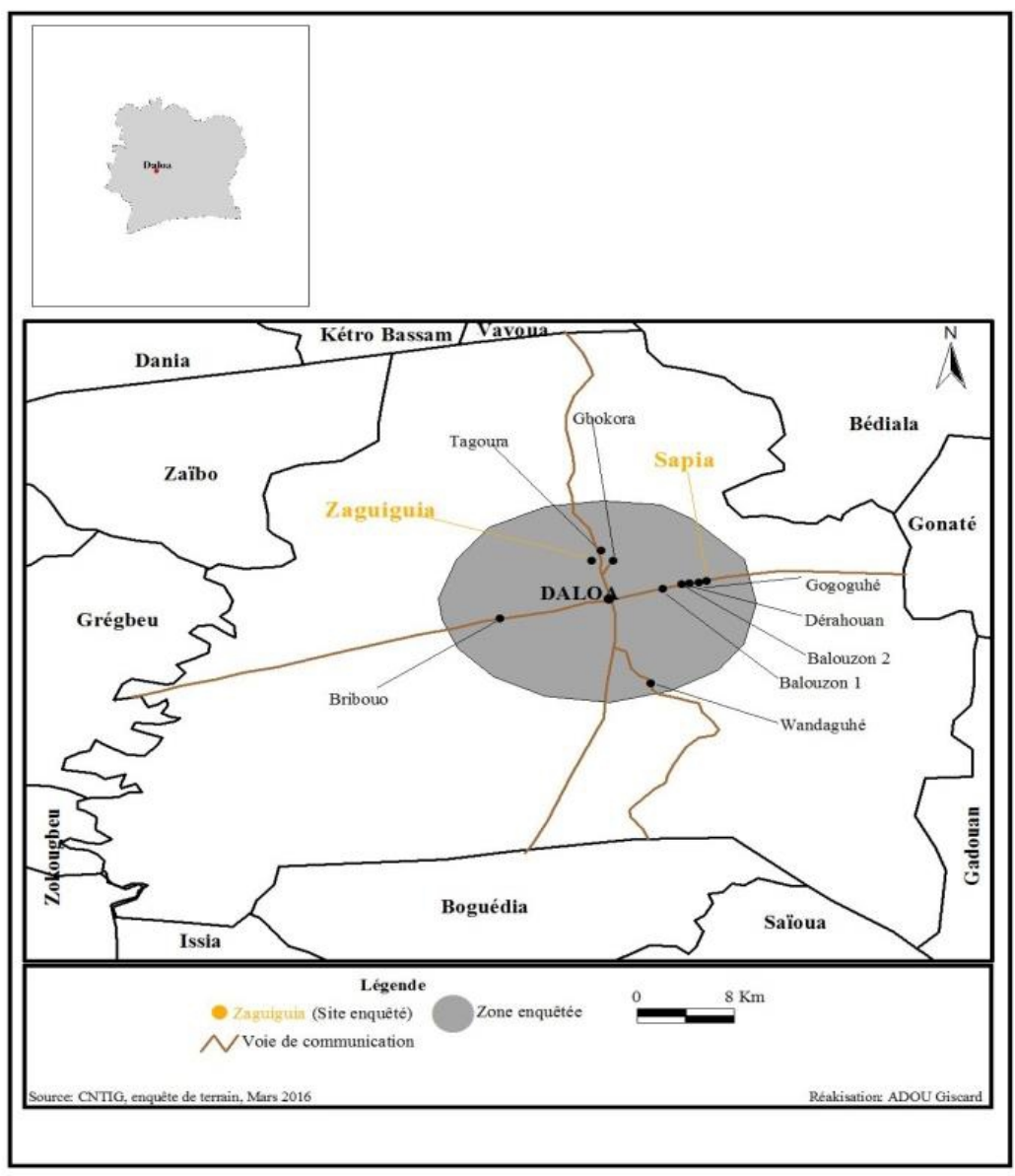

\section{Analyse des données}

La méthodologie utilisée repose sur la documentation et l'observation directe soutenue par les enquêtes de terrain.

La documentation consultée est constituée en partie de fonds de cartes cartographiques et de documents administratifs mis à notre disposition par le Ministère de la Construction et de l'Urbanisme, la Mairie, la Préfecture et la Sous-préfecture. Les fonds de cartes ont servi à mieux circonscrire notre zone d'étude. Parmi les différentes données disponibles, nous avons privilégié celles en rapport avec le phénomène étudié, complétées à la suite par nos enquêtes.

L'enquête de terrain a été menée dans deux villages communaux situés dans le front d'extension de la ville : Sapia et Zaguiguia.

Au total, 100 ménages (en raison de 50 par village) ont été enquêtés à l'aide d'un questionnaire. Il a été complété par un guide d'entretien adressé aux autorités coutumières desdites entités villageoises. Parallèlement aux 
enquêtes menées dans les villages, d'autres cibles d'enquête ont été retenus pour des entretiens. Il s'agit notamment de la Direction régionale du Ministère de la Construction et de l'Urbanisme, du service technique de la Mairie, de la Préfecture et de la Sous-préfecture.

\section{Résultats et discussion}

\section{De l'aperçu de l'étalement de la ville de Daloa}

L'étalement de Daloa est dû aux facteurs géographique, politique, économique et démographique.

\section{Une extension spatiale sous le poids de facteurs géographique et politique}

Née de la fusion des villages de Lobia, Labia, Gbeuliville et Tazibouo, situés en bordure des pistes Nord-Sud et Est-Ouest, Daloa est avant tout le territoire des populations autochtones Bété. C'est donc sous la férule du régime foncier traditionnel que les prémices de l'extension spatiale de la ville ont été posées par l'administration coloniale. Les toutes premières matérialisations ont été le fait de la réalisation de voies de communication réticulaires entre cet espace et un ensemble de villes (Kipré, 1985). Le réseau viaire de maille orthogonal s'est par la suite développé tout en facilitant les dessertes à l'intérieur de cette ville propice au transport. Mais, le développement de ce réseau a été favorisé voire accéléré par les échanges entre les villes de la région, du territoire national et même de la sous-région (Alla, 1991 ; Koffi, 2010). Par ailleurs, sa position de ville carrefour a facilité son passage de poste colonial à celui de poste administratif. Les effets d'une telle transformation sont marqués par la consommation d'espace à travers l'implantation d'une série d'équipements qui renforcent du coup le poids administratif de la ville (Alla, 1991).

\section{Une dynamique spatiale favorisée par les activités économiques}

L'essor économique de la ville est lié à la présence de différentes activités économiques qui ont contribué à son développement. La première de ces activités a été le commerce de la cola qui, au $19^{\text {ème }}$ siècle, a fait de la ville un centre important de négoce tant dans la région que dans la sousrégion (Alla, 1991). Cette activité économique a attiré de nombreuses populations d'origines diverses à Daloa. Cela a conduit à la naissance de quartiers dont la dénomination repose sur des bases ethniques (Alla, 1991). A partir de 1950, les cultures du café et du cacao prennent le relais, à un moment où la cola déclinait. Le succès économique que connaissent les deux cultures a également été une source indéniable d'attraction de populations. Les conséquences en ont été la création de nouveaux équipements ainsi que de nouveaux quartiers (Alla, 1991 ; Koukougnon, 2012). Aux activités 
agricoles, se sont ajoutées celles liées à l'industrie du bois, le tout contribuant non seulement au développement spatial mais également au croît démographique de Daloa, modelant ainsi l'espace urbain (Alla, 1991 ; Yao, 2014).

\section{Un accroissement spatial sous l'effet de la poussée démographique}

La croissance de la population de Daloa est en rapport avec le rythme de l'activité économique. C'est véritablement à partir de la hausse du prix du cacao dans les années 1950 que Daloa est devenue un centre urbain attractif. Daloa devient de par l'effectif de sa population (35 000 habitants), la troisième ville de Côte d'Ivoire après Abidjan (350 000 habitants) et Bouaké, avec 85000 habitants (Kipré, 1985 ; Alla, 1991). Entre 1980 et le début des années 1990 le rythme de peuplement de la ville connaît une augmentation (6,4\% par an). Corrélativement, la consommation d'espace devient impressionnante. Au sortir de cette période, la commune s'étend désormais sur une superficie de 1234 ha avec une population de 122933 habitants (Alla, 1991). Cette consommation d'espace n'est pas sans conséquences sur les rapports fonciers. Les villages de Lobia et de Tazibouo expriment en effet leur mécontentement par la revendication de lots de compensation en raison de lotissements effectués sur leurs finages pour l'extension de la ville (Alla, 1991). Aujourd'hui, le périmètre communal de la ville de Daloa s'étend sur près de 8000 ha (RGPH, 2014 ; Mairie, 2016).

$\mathrm{Au}$ total, l'extension spatiale de la ville de Daloa n'est pas récente. C'est un phénomène qui tire ses origines dans les années 1950. Toutefois, les rythmes de croissance de l'espace urbain ont été marqués par deux phases. La première, qui s'est faite de 217,75 ha en 1955 à 1340 en 1988, soit un taux d'extension de 5,66\% (Alla, 1991). La seconde phase, de 1988 à nos jours, est tout aussi dominée par une croissance spatiale forte. Le taux annuel de $6,56 \%$ est nettement supérieur au taux de croissance démographique de 1,93\% (RGPH, 1998 ; 2014). Cette extension galopante de la ville n'est pas sans effets sur les rapports fonciers avec les villages périphériques dont notamment Sapia et Zaguiguia.

\section{Ville de Daloa-villages périphériques : état des rapports fonciers, évolution de la situation}

Des rapports fonciers reposant sur la collaboration des acteurs jusqu'en 1980

L'urbanisation de Daloa s'est déroulée sous le double appui du droit de propriété moderne et du droit coutumier traditionnel. C'est donc dans ce contexte de coexistence de deux régimes juridiques en matière foncière que les différents plans d'aménagement de Daloa sont exécutés (Alla, 1991). Dans l'ensemble, le développement de la ville s'est opéré à travers deux 
approches. La première, sous la forme de négociations entre les chefs de villages et l'administration coloniale. La seconde approche, est une opération administrative qualifiée d'utilité publique par laquelle l'administration (tant coloniale que postcoloniale) se rend propriétaire des terres. Lesdites pratiques ont conduit à des tensions foncières qui n'ont pas pour autant stoppé la dynamique d'aménagement de la ville (Alla, 1991). Fort des expériences de la période coloniale, les autorités ivoiriennes décident à l'indépendance de faire des déguerpissements des villages, une exception. Le déguerpissement s'opère désormais lorsque le village est sur l'emprise d'une infrastructure publique ou d'un projet d'intérêt commun (Alla, 1991 ; Dembélé, 1997). L'enjeu d'une telle stratégie est la production et l'organisation cohérente de la ville, conformément aux options urbanistiques inscrites dans les documents d'urbanisme (Ouattara, 1999). Il n'est pas rare de voir en effet, certains propriétaires coutumiers qui, du fait de l'avancée du front urbain et de la menace que cela fait peser sur leurs terrains, de procéder à la création d'habitats spontanés ou de s'opposer ouvertement aux lotissements initiés (Alla, 1991). Pour sortir de cette contrainte, l'Etat opte pour la neutralisation des forces coutumières, comme un moyen dont il ne saurait se priver. Il s'agit de disposer de l'assiette foncière permettant de trancher avec les pratiques du passé marquées par le recours à la force (Gouamené et al., 2016). Il est question désormais d'associer les populations coutumières à travers des négociations directes avec celles-ci, en vue de redéfinir avec elles, les conditions de libération des terrains sous leur emprise (Yapi-Diahou, 1994).

\section{Des rapports fonciers tendus à partir de 1980}

En 1980, 1'Etat décide de la politique de communalisation, qui se veut un développement participatif autour d'initiatives locales de développement dont les collectivités territoriales sont présentées comme les artisans. Cette politique recadre non seulement les rapports entre populations et administrations en général mais encore entre populations coutumières et administration centrale en particulier à Daloa. En effet, des incidents ont eu lieu en 1978 à Daloa suite à des désaccords entre l'administration centrale et les populations rurales. Dans les villages de Lobia et de Tazibouo par exemple, des contestations ont eu lieu au sujet de lots de compensation, en raison de lotissements effectués sur les finages villageois.

$\mathrm{Au}$ regard des expériences passées, la nouvelle orientation des rapports fonciers entre communautés coutumières et pouvoir central se déroule dans un environnement de dynamique plus revendicative (Affou, 1997). Ainsi, face à l'empiètement de leur terre par la ville et les promesses non tenues par les autorités selon les communautés villageoises ; l'espace devient un nouvel enjeu au centre duquel de nouveaux rapports se 
définissent. La situation qui prévaut n’épargne aucunement les villages de Sapia et de Zaguiguia.

\section{Sapia et Zaguiguia : deux espaces périphériques de Daloa au centre de nombreuses tensions foncières}

Des tensions foncières et de leur intensification

Les problèmes sociaux dont découlent les tensions foncières ont toujours existé dans les sociétés paysannes en Afrique. Leurs amplifications sont nées des mutations qui se sont opérées dans lesdites sociétés (Zunon, 1981 ; Ibo, 1997). Les villages de Sapia et Zaguiguia ne sont pas restés en marge de cette dynamique d'ensemble. Les registres de l'administration centrale mis à notre disposition et les entretiens réalisés avec les populations dans les villages enquêtés ont confirmé les faits. La progression graduelle de l'importance des tensions foncières dans les villages atteste en effet de l'ampleur du phénomène (tableau 1).

Tableau 1 : Cas de tensions foncières dans les villages enquêtés

\begin{tabular}{|c|c|c|c|c|c|c|c|c|c|c|c|c|c|c|c|}
\hline \multirow[t]{2}{*}{ Localités } & \multicolumn{15}{|c|}{ Date des tensions foncières } \\
\hline & \begin{tabular}{l}
+ \\
$\infty$ \\
\hdashline
\end{tabular} & $\stackrel{\approx}{\Omega}$ & ஃ̊ & જ̆ & ్ㅗㅇ & 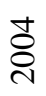 & 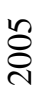 & ஓి & 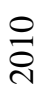 & $\overline{\vec{d}}$ & 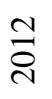 & $\frac{m}{\stackrel{\sim}{c}}$ & 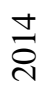 & $\stackrel{n}{\stackrel{n}{\sim}}$ & $\frac{0}{\stackrel{0}{\sigma}}$ \\
\hline Sapia & 0 & 0 & 1 & 0 & 0 & 0 & 1 & 0 & 0 & 1 & 1 & 2 & 0 & 8 & 3 \\
\hline Zaguiguia & 1 & 1 & 0 & 1 & 1 & 1 & & 1 & 1 & 0 & 2 & 3 & 5 & 4 & 3 \\
\hline TOTAL & 1 & 1 & 1 & 1 & 1 & 1 & 1 & 1 & 1 & 1 & 3 & 5 & 5 & 12 & 6 \\
\hline
\end{tabular}

Sur la période 1980-2000, les cas de tensions foncières connaissent une faible intensité soit un taux d'accroissement moyen de 7,18\% par an. Puis, une quinzaine d'années plus tard, les tensions foncières ont doublé d'intensité avec un taux d'accroissement moyen de 14,91\% par an. La multiplication des tensions comme l'indique le tableau, traduit les enjeux qui se nouent autour du foncier dans les villages. En somme, l'espace devient un nouvel enjeu avec au centre, les compétitions que se livrent les acteurs pour l'occupation du sol. Les tensions qui en découlent se règlent autant dans les villages qu'à l'extérieur de ceux-ci. Toutefois, les motifs desdites tensions sont tout aussi divers que variés.

\section{Sources des tensions foncières dans les villages}

Cet aspect de notre étude permet de comprendre les dynamiques foncières qui ont cours et les enjeux qu'elles suscitent dans le cadre de l'urbanisation au niveau des espaces périurbains (Yapi-Diahou, 1991). Les villages de Sapia et de Zaguiguia illustrent les mutations qui ont cours. Il ressort de notre enquête que les sources des tensions dans ces villages sont multiples et variées (tableau 2). 
Tableau 2 : Motifs des tensions foncières selon les enquêtés

\begin{tabular}{|c|c|c|c|c|c|c|}
\hline Localités & \multicolumn{6}{|c|}{ Motifs } \\
\cline { 2 - 7 } & $\begin{array}{c}\text { Revendication } \\
\text { de droit de } \\
\text { propriété }\end{array}$ & $\begin{array}{c}\text { Opposition } \\
\text { à la vente } \\
\text { d'un bien } \\
\text { collectif }\end{array}$ & Empiètement & $\begin{array}{c}\text { Contestation } \\
\text { du mode de } \\
\text { gestion des } \\
\text { terres }\end{array}$ & $\begin{array}{c}\text { Purge de } \\
\text { droits } \\
\text { coutumiers }\end{array}$ & $\begin{array}{c}\text { Non- } \\
\text { respect } \\
\text { de } \\
\text { contrat }\end{array}$ \\
\hline Sapia & 11 & 1 & 4 & 1 & 0 & 0 \\
\hline Zaguiguia & 17 & 1 & 3 & 1 & 2 & 1 \\
\hline TOTAL & 28 & 2 & 7 & 2 & 2 & 1 \\
\hline
\end{tabular}

Source : notre enquête de terrain, 2016

L'analyse générale du croisement des motifs des tensions autour de la terre révèle comme forme dominante, la revendication de droit de propriété $(66,67 \%)$; suivent après, l'empiètement $(16,67 \%)$; l'opposition à la vente d'un bien collectif $(4,76 \%)$; la purge des droits coutumiers $(4,76 \%)$; la contestation du mode de gestion des terres $(4,76 \%)$ et le non-respect de contrat $(2,38 \%)$. L'importance des motifs des tensions foncières relatifs à la revendication de droit de propriété $(66,66 \%)$ témoigne de la logique du développement de la propriété individuelle dans le contexte de mutations et de transformations du régime coutumier traditionnel qui prévaut. Le nouveau statut de la terre marqué en effet par le changement économique et la compétition pour son appropriation conduisent à des tensions autour de la propriété foncière.

La terre est perçue comme un bien individuel ou un bien privé. Désormais, sa gestion échappe au chef de famille ou au chef de clan. De telles situations sont à l'origine des tensions foncières observées dans les villages. La question de l'appropriation privée de la terre dans la survenance des tensions foncières reste essentielle (Feeny, 1988 ; Koffi, 2007). En ce qui concerne particulièrement la ville de Daloa, les tensions relatives à l'empiètement découlent de deux facteurs. Le premier, est relatif à l'afflux de populations que la ville a accueillies relativement à la crise militaropolitique, participant ainsi à la hausse de la demande de logements. Le second facteur est dû à la rareté des terres cultivables que cette affluence de populations a occasionnée (Mafou et al., 2016). La pénurie a provoqué non seulement la vente multiple des terres à différents acquéreurs voire l'installation à titre temporaire de populations. Il en est résulté une compétition qui a conduit à des difficultés de délimitation des parcelles. On assiste alors à des tensions entre populations autochtones $(28,58 \%)$ et entre autochtones et allogènes $(71,42 \%)$. Dans l'ensemble, les conflits fonciers sont liés non seulement au jeu de la diversité des logiques des acteurs mais également au jeu de pouvoir auquel se livrent ceux-ci dans le système social (Chauveau, 1998 ; Raffestin et al., 2001). Pour tout dire, les pratiques 
foncières des acteurs sur les règles coutumières sont déterminées suivant des logiques de rapports de force.

S'agissant de la purge des droits coutumiers dans le cadre du développement urbain de Daloa, les différentes autorités tant coloniales que postcoloniales, ont passé des accords avec les populations autochtones. Ces conventions permettent aux pouvoirs publics de prendre possession des terres en indemnisant les détenteurs coutumiers (Yapi-Diahou, 1981 ; Alla, 1991). Dans la pratique, ces opérations se sont toujours déroulées avec assez de heurts. Les entretiens réalisés avec les autorités municipales ont permis de noter que les villages de Gbeuliville, Lobia, Labia, Gbokora, Tazibouo et certaines familles (autochtones comme allogènes) remettent en cause le principe. L'incorporation des terres des villages pour l'aménagement de la ville, laisse des populations "sans terre" et des communautés coutumières insatisfaites des indemnisations perçues.

Contrairement aux villages Ebrié qui revendiquent des lots entièrement lotis par l'Etat, et mis à la disposition de la collectivité comme complément aux indemnités jugées insuffisantes, certaines familles revendiquent dans le cas de Zaguiguia, des terrains appartenant déjà à l'Etat ou à des particuliers. C'est alors qu'elles procèdent à leur morcellement.

Au total l'étalement de la ville de Daloa a donné naissance à des tensions foncières dans les villages périphériques. Aussi, les sensations produites par ce phénomène imposent des stratégies que développent les populations rurales.

\section{Perception de l'étalement urbain et stratégies développées par les populations}

Les populations des villages périphériques face à l'étalement émettent des idées, expriment des sentiments mais développent également des stratégies.

\section{Perception de l'étalement urbain \\ L'étalement urbain : un sentiment d'angoisse}

La perception de l'étalement urbain repose sur les représentations que l'ensemble des acteurs impliqués dans les tensions foncières se font de l'espace. Bailly (2001) définit la représentation comme étant « une création sociale ou individuelle de schémas pertinents du réel dans le cadre d'une idéologie ; elle consiste soit à évoquer des objets en leur absence, soit, lorsqu'elle double la perception en leur présence, à compléter la connaissance perceptive en se référant à d'autres objets non actuellement perçus. » Ce faisant, le groupe de sentiments développés porte la marque de l'angoisse développée par les communautés villageoises face au fait urbain, à travers les revendications territoriales, la purge de droits coutumiers ou le 
climat d'insécurité qui s'instaure. Les populations de Zaguiguia expriment ce désarroi en ces mots : "Avec l'avancée de la ville... l'Etat, à travers la Marie, nous arrache nos terres pour ses projets de développement. Nous sommes impuissants face à ces pratiques ». Ces propos traduisent en réalité la mise en place d'une diversité de logiques des acteurs qui reposent essentiellement sur des rapports de force. L'ensemble des inquiétudes développées par les populations à travers ces pratiques se matérialise par la disparition de leurs plantations voire de l'abandon de l'activité agricole. Par ailleurs, l'argument développé rejoint la prise en compte de leurs droits ancestraux en rapport avec leur patrimoine (Hoffmann, 2002). L'image née de la perception se réfère de ce fait, à un environnement matériel qui perturbe l'existence quotidienne du groupe social (Debardieux, 2001).

\section{L'étalement urbain : une opportunité à saisir}

Le second groupe d'arguments développés par les populations, présente l'étalement urbain comme bénéfique, une opportunité, voire une " bonne chose ». Le processus de représentation constitutif de la relation que les populations établissent avec le phénomène est à la fois sensoriel et pratique (Debardieux, 2001). En somme, les aspects positifs de l'étalement urbain sont présentés sous différents angles selon la tournure des échanges et les appréciations des acteurs interrogés. Les propos de la représentante des femmes de Sapia expliquent mieux la situation qui prévaut : « La ville a pris toutes nos terres cultivables donc nous sommes obligées de venir vendre et de mener des activités différentes de l'agriculture». Ces propos témoignent de la capacité d'adaptation des populations à faire face à ce phénomène. Toutefois, les thèses avancées pour la plupart des acteurs interrogés, indiquent que l'extension de la ville de Daloa a permis aux villages de diversifier leurs activités économiques, d'étendre leurs superficies, de dynamiser leurs économies et d'être plus accessibles. En somme, cela favorise le renouvellement des contacts sociaux (Antoni, 2014 ; Mafou et al., 2016). Par ailleurs, cette perception n'est pas différente de celle de Hilgers (2008) notamment au Burkina Faso. Il souligne en effet les apports bénéfiques du fait urbain en termes d'accessibilité, d'amélioration des conditions de vie des populations dans les espaces ruraux.

\section{L'étalement urbain : un phénomène ambivalent}

Le troisième groupe d'arguments apparait, par contre, sous un double aspect. Le premier aspect sur les formes de régulation de la société exprimées par les jeunes. Et le second aspect sur la cherté de la vie. Pour les jeunes, le mode de gestion des terres par les aînés ne correspond pas aux réalités d'une société en mutation. Ils sont à la recherche d'un équilibre social par rapport à la gestion des terres mais aussi, en quête d'une sorte " 
d'accommodement » dans les modes de succession dans un contexte marqué par la rareté foncière. A cet effet, les propos du représentant des jeunes du village de Zaguiguia expliquent mieux le climat qui prédomine :

Du fait de l'avancée de la ville, les chefs de lignages et de familles morcellent et vendent les terres sans le consentement des membres de la famille et sans penser aux générations futures. Or, aujourd'hui les temps sont difficiles et la terre reste notre seul espoir en termes de création de petites exploitations agricoles. En effet, la probabilité de trouver un emploi en ville reste incertaine.

Ce discours résume en partie une remise en cause de la légitimité dont disposent les institutions ou les autorités. La plupart des jeunes évoquent en effet un argument basé sur le droit à la parole dans une société villageoise traversée par plusieurs courants de changements (Gouamené, 2013). De telles situations conduisent à la longue à la fragmentation de l'autorité aussi bien de ceux qui les incarnent que des institutions (Chauveau, 1997/1998 ; Raffestin et al., 2001). Quant aux arguments en lien avec la cherté de la vie, ils sont en rapport avec les nouveaux modes de vie introduits par les coûts des déplacements des ménages (par l'emprunt de taxis communaux) et les nouvelles formes de consommation qui s'imposent de plus en plus aux populations. En somme, les habitants des villages enquêtés ne s'approprient pas le nouveau modèle social dans lequel ils s'intègrent (Beaujeu-Garnier, 2006 ; Mafou et al., 2016).

De façon générale, la croissance effrénée de la ville de Daloa a confiné bon nombre de villages de la commune de Daloa sur des surfaces réduites annihilant de ce fait leurs possibilités d'extension. Aujourd'hui, la colonisation des espaces ruraux par le fait urbain montre la reconversion des populations de ces espaces villageois qui sont devenues en parties citadines. Aussi, la question qui transparait est de savoir les stratégies mises en place par les populations face à l'étalement urbain.

\section{Les stratégies développées par les populations}

Face à l'étalement urbain, les populations des villages périphériques diversifient leurs activités économiques et se solidarisent par la création d'associations à base identitaire.

\section{L'intégration économique}

Dans son extension, la ville de Daloa a conduit à la fin progressive de l'agriculture dans les villages périphériques. Les populations de ces entités spatiales ont en contrepartie saisi les opportunités offertes par l'économie urbaine. A ce sujet, les stratégies intégratives au processus d'urbanisation développées par les villages se manifestent surtout sur le plan économique à 
travers le morcellement et la vente des terres. A cet effet, les propos du chef de terre du village de Sapia traduisent mieux la situation qui a cours : « les populations détruisent leurs champs en faisant lotir leurs parcelles à cause de l'avancée de la ville ». Face à l'avancée de la ville les terres agricoles prennent de la valeur en raison des programmes de lotissement dont les retombées financières sont considérables pour leurs détenteurs. Le $\mathrm{m}^{2}$ peut se négocier entre 1000 F CFA et 1500 F CFA. Ainsi, la vente d'une parcelle de $500 \mathrm{~m}^{2}$ peut rapporter une somme comprise entre 500000 FCFA et 1000 $000 \mathrm{~F}$ CFA. Des montants alléchants pour détourner les populations des activités agricoles.

A côté de la vente des terres, les populations s'intéressent également aux activités liées aux commerces pour lutter dans une certaine mesure contre le chômage, la délinquance juvénile et la pauvreté. De telles activités économiques ne sont pas différentes de celles que pratiquent les autochtones Ebrié dans le cas d'Abidjan (Boti-Bi, 2008 ; Oura et al., 2016).

\section{La création d'associations locales}

A l'opposé des stratégies d'intégration économique développées, une autre stratégie est également mise en œuvre par les villages notamment sur le plan sociopolitique. Il s'agit pour les populations Bété de créer des associations à base identitaire sur le modèle Ebrié en réponse aux enjeux urbains. Cependant, comme le souligne Gnabéli (2008), les associations qui naissent en pays Bété à la différence de celles des villages Ebrié, ont peu d'emprise sur les rapports des villageois à la ville. Le problème de l'aménagement des villages en pays Bété se présente sous des contours encore flous à la différence des villages Ebrié. Dans l'ensemble, la politique d'exception mise en place par les gouvernements d'alors en rapport avec le maintien ou le déguerpissement des villages, a légitimé la création de modes d'organisation et de gestion qui échappent au contrôle des pouvoirs publics. A cet effet, les villages conservent une bonne partie de leurs règles d'organisation et de gestion (Oura, 2013). Dans les faits, le statut 'd'extraterritorialité', dont ils se prévalent leur confère certaines franchises vis à vis de l'administration communale (Gouamené et al., 2016; Mafou et al., 2016). A certains égards, l'intégration des villages au processus d'urbanisation va se présenter comme un droit ou comme une exception. A ce sujet, la question liée à l'aménagement des entités villageoises ne trouve pas de solution de manière définitive du fait des tensions foncières permanentes (Alla, 1991). De telles pratiques ne sont pas différentes de celles observées dans les villages Ebrié, au sud de la Côte d'Ivoire (Oura, 2013 ; Gouamené et al., 2016). 


\section{Conclusion}

L'étalement de la ville de Daloa s'est fait sous l'effet de trois principaux facteurs. Ce sont notamment les facteurs géographiques et politiques, les facteurs liés aux activités économiques et ceux en rapport avec la croissance démographique. Dans cette extension spatiale, les rapports dans le domaine foncier avec les villages périphériques ont évolué selon différentes variantes. Si au départ, ces rapports fonciers étaient basés sur la collaboration, ils ont été par la suite tendus au fil des ans en raison des enjeux autour de la terre. L'extension de la ville a en effet non seulement réduit les finages des villages mais également changé le rapport de l'homme à l'espace. Dans l'ensemble, le jeu foncier est caractérisé par un ensemble de règles et de pratiques qui portent la marque de communautés traversées par une série de changements notamment au plan sociopolitique, économique voire de leur aménagement. La perception de l'étalement urbain à travers les tensions foncières est en effet influencée non seulement par les conditions socioéconomiques des populations, mais aussi par la perturbation du fonctionnement de la structure sociale au sein des villages. Face aux changements qui ont cours dans les rapports ville de Daloa-villages périphériques, les populations développent non seulement des stratégies économiques, politiques mais également organisationnelles.

\section{References:}

1. ADE Kacou, 2008, Production de l'espace périurbain de Bingerville/Cocody. Mémoire de Maîtrise. Université deCocody, 113 p.

2. AFFFOU YAPI Simplice, 1997, « Renforcement des organisations paysannes et progrès agricole : obstacles ou atouts pour le progrès agricole », dans B. Contamin et H. Memel-Fotê (Éds), Le Modèle Ivoirien en Questions. Paris, Karthala, pp. 555-571.

3. ALLA DELA André, 1991, Dynamisme de l'espace péri-urbain de Daloa, étude géographique, Thèse de doctorat 3ème cycle, Abidjan, Université de Cocody, IGT, 453 p.

4. ANTONI Jean-Philippe, 2014, «L'étalement urbain ». La France en villes, [En ligne] 164-176, mis en ligne le 20 octobre 2014, consulté le 20 décembre 2016. URL: https://hal.archivesouvertes.fr/hal-01075776

5. BEAUJEU-GARNIER Jacqueline, 2006, Géographie urbaine, Paris, Armand Colin, 349 p.

6. BAILLY Antoine, 1999, « Pour un développement social urbain durable » sous la direction de BAILLY Antoine et HURIOT JeanMarie, Villes et Croissance. Paris, Anthropos, pp. 259-273. 
7. BAILLY Antoine et BEGUIN Hubert, 2001, Introduction à la géographie humaine, Paris, Armand Colin, 216 p.

8. BOTI-BI Trayié Cyril, 2008, « Les tentatives de confiscation des services urbains par le pouvoir coutumier à Abidjan », dans S. Bredeloup, B. Bertoncello et J. Lombard (Éds), Abidjan,Dakar : des villes à vendre? (La privatisation made in Africa des services urbains). Paris, L'Harmattan, pp. 115-129.

9. CHAUVEAU Jean-Pierre, 1997, Jeu foncier, institutions d'accès à la ressource et usage de la ressource : une étude de cas dans le Centre-ouest ivoirien, In Le Modèle Ivoirien en Questions. Crises, ajustement et recompositions, Paris, Karthala, pp. 325-360.

10. CHAUVEAU Jean-Pierre, 1998, « Quelle place donner aux pratiques des acteurs ? 》In Quelles Politiques Foncières pour l'Afrique Noire? , Karthala, Coopération Française, pp. 36-39.

11. DEBARDIEUX Bernard, 2001, « Les problématiques de l'image et de la représentation en géographie ", In Les Concepts de la Géographie Humaine, Paris, Armand Colin, pp. 199-211.

12. DEMBELE Ousmane, 1997, « Le modèle d'urbanisme ivoirien face à la crise économique ", dans B. Contamin et H. Memel Fotê (Éds), Le Modèle Ivoirien en Questions. Paris, Karthala, pp. 483-511.

13. DIBY Kouakou Martin, 2012, « Structures politiques villageoises et revendications d'intégration économique : cas des Ebrié de Yopougon ». Revue de Géographie de Lomé, n 8, pp. 38-47.

14. FEENY David, 1988, «The development of property rights in land: a comparative study», In Bates R. (éd), Toward a political economy of development, Berkeley, Los Angeles et Londres, University of California, Press, pp. 272-299.

15. GNABELI Roch Yao, 2008, « La production d'une identité autochtone en Côte d'Ivoire ». Journal des anthropologues [En ligne], 114-115 |, mis en ligne le 01 décembre 2009, consulté le 05 juin 2016. URL : http://jda.revues.org/326

16. GOUAMENE Didier Charles, 2013, Espaces urbains et conflits fonciers dans l'agglomération d'Abidjan: cas des communes de Cocody et de Treichville, Thèse de doctorat enGéographie, Université Abidjan-Cocody, 382 p.

17. GOUAMENE Didier Charles, ATTA KOFFI Lazare et GOGBE Téré, 2016, « Aménagement urbain et logiques intégratives à Abidjan : cas des villages Ebrié de la commune de Cocody », Revue des Langues, Lettres et Sciences de l'Homme et de la Société, Université de Kara-Togo, n ${ }^{0} 1$, pp. 487-512 
18. HILGERS Mathieu, 2008, « Politiques urbaines, contestation et décentralisation : lotissement et représentation au Burkina-Faso ». Autrepart, $\mathrm{N}^{0} 47$, IRD, pp. 209-225.

19. HOFFMANN Odile, 2002, « L'émergence de la notion de patrimoine dans le cadre de revendications identitaires et territoriales (Pacifique colombien) », dans M-C. Cormier-Salem., D. JuhéBeaulaton., J. Boutrais « et al »(Éds), Patrimonialiser la nature tropicale (Dynamiques locales, enjeux internationaux). Paris, pp. 191-213.

20. IBO Jonas, 1997, « Litiges de forêts dans la région de Sassandra. Quelles implications ? », dans B. Contamin et H. Memel Fotê (Éds), Le Modèle Ivoirien en Questions. Paris, Karthala, pp. 379-391.

21. KIPRE Pierre, 1985, Villes de Côte d'Ivoire 1893-1940 : économie et société urbaine, tome 2, Abidjan, N.E.A, 290 p.

22. KOFFI Adjoba Marthe, 2007, Mutations sociales et gestion de l'espace rural en pays Ebrié (SUD-EST de la Côte d'Ivoire), Thèse de Doctorat en Géographie, Université Paris I Panthéon-Sorbonne, $412 \mathrm{p}$.

23. KOUKOUGNON Wilfried., 2012, Milieu urbain et accès à l'eau potable : cas de Daloa (centre-ouest de la Côte d'Ivoire), Thèse de doctorat en Géographie, Université Abidjan-Cocody, 365 p.

24. LE ROY Etienne, KARSENTY Alain et BERTRAND Alain, 1996, La sécurisation foncière en Afrique (pour une gestion viable des ressources renouvelables). Paris, Karthala, 389 p.

25. MAFOU Kouassi Combo, GOHOUROU Florent et OURA Kouadio Raphael, 2016, « Croissance démographique et consommation des espaces périphériques de la ville de Daloa (centreouest ivoirien) ». Revue Internationale de Recherches et d'Etudes Pluridisciplinaires, Centre Lyonnais sur les identités professionnelles et sociales, Université Lumière Lyon II, pp. 7-21.

26. OUATTARA Issa, 1999, «Urbanisme autochtone et urbanisme étatique : Antagonisme ou complémentarité ». Cahiers Nantais $N$ 51, pp. $7-17$

27. OURA Kouadio Raphael, 2013, «Urbanisation de la métropole abidjanaise et la mise en minorité des autochtones Ebrié », Cinq Continents, Revue Roumaine de Géographie, Volume 3 / Numéro 8, pp. 150-168.

28. OURA Kouadio Raphael, KOUAKOU Louise Aya et GOUAMENE Didier Charles, 2016, « Urbanisation et pression foncière dans les campagnes d'autochtones Ebrié d'Abidjan ». Revue Africaine de Criminologie, $\mathrm{N}^{\circ} 19$, EDUCI, Université F. H. B. pp. 7991. 
29. PAULET Jean-Pierre, 2000-Géographie urbaine, Paris, Armand Colin, $310 \mathrm{p}$.

30. RAFFESTIN Claude et BARAMPAMA Angelo, 2001, « Espace et pouvoir », In Les Concepts de la Géographie Humaine, pp. 63-71.

31. ZUNON GNOBO Julien, 1981, Les échanges dans la région de Daloa du milieu du 19eme siècle à 1936, Paris, Thèse 3e cycle, 554 p.

32. YAO Kouassi Ernest, 2014, L'impact des unités industrielles de transformation du bois sur le développement urbain à Daloa, Thèse unique de doctorat de géographie, Université Félix HouphouëtBoigny d'Abidjan-Cocody, IGT, $291 \mathrm{p}$.

33. YAPI-DIAHOU Alphonse, 1981, Étude de l'urbanisation de la périphérie d'Abidjan, l'urbanisation de Yopougon, Thèse de Doctorat de $3^{\text {eme }}$ Cycle, Université Toulouse le Mirail, 322 p.

34. YAPI-DIAHOU Alphonse, 1991, " Les détenteurs coutumiers, les citadins et l'État dans la course pour l'accès au sol urbain à Abidjan », In contribution à la connaissance d'un droit intermédiaire dans les villes d'Afrique de l'Ouest, CNRST -IFV, Ostom, pp. 11-84.

35. YAPI-DIAHOU Alphonse, 1994, Politiques urbaines en Côte d'Ivoire son impact sur l'évolution des formes d'habitats non planifié : exemple de l'agglomération d'Abidjan, Thèse de Doctorat d'Etat, Paris, $718 \mathrm{p}$. 